\title{
Reporter gene HEK 293 cells and WNT/Frizzled fusion proteins as tools to study WNT signaling pathways
}

\author{
Larisa Ring, Iris Peröbner, Marisa Karow, Marianne \\ Jochum, Peter Neth and Alexander Faussner* \\ Abteilung für Klinische Chemie und Klinische Biochemie, \\ Ludwig-Maximilians-Universität, Nussbaumstrasse 20, \\ D-80336 München, Germany \\ *Corresponding author \\ e-mail: alexander.faussner@med.uni-muenchen.de
}

\begin{abstract}
WNT/Frizzled receptor (FZD) signaling pathways are pivotal for physiological and pathophysiological processes. In humans, the complexity of WNT/FZD signaling is based on 19 WNTs, 10 FZDs and at least two (co)receptors (LRP5/6) mediating supposably four different signaling cascades. The detailed investigation of the specific function of the different initiating components is primarily hampered by the lack of most WNT proteins in a purified form. Therefore, we constructed and examined a chimeric protein of WNT3a and FZD4 as a suitable approach to overcome this obstacle for future studies of the specificity of other WNT/FZD combinations. Furthermore, we produced four different reporter HEK 293 cell lines to quantify the induced activation of the proposed signaling cascades, the $\beta$-catenin-, the NFAT-, the AP-1- and the CRE-regulated pathways. The chimera WNT3aFZD4 efficiently induced $\beta$-catenin-mediated luciferase activity. This activity was increased 40-fold compared with basal when LRP6 was stably cotransfected, proving that the chimera WNT3aFZD4 can also interact efficiently with LRP6. Our results demonstrate that the approach of using reporter gene cell lines in combination with WNT/FZD chimeras is efficient to study the $\beta$-catenin-mediated pathway and should also allow clarifying the specificity of WNT/FZD combinations in the activation of the other pathways.
\end{abstract}

Keywords: activator protein 1 (AP-1); cyclic adenosine monophosphate response element (CRE); G-protein-coupled receptor (GPCR); nuclear factor of activated T cells (NFAT); $\mathrm{T}$ cell factor/lymphoid-enhanced factor (TCF/LEF).

\section{Introduction}

The importance of the physiologically relevant WNT/Frizzled receptor (FZD) signaling pathways becomes particularly apparent when they are dysregulated, often leading to severe diseases, such as cancer, osteoporosis and degenerative illnesses (Logan and Nusse, 2004). Regarding the initiating components of the respective pathways, a family of globular proteins, commonly referred to as WNTs (an acronym combining wingless and int1), has been identified as main ligands of the FZDs and their coreceptors within the past 25 years (Klaus and Birchmeier, 2008), indicating the great complexity of the WNT/FZD signaling system. The WNT proteins (19 members in humans) belong to a large, evolutionarily conserved family of extracellular signaling ligands, which are secreted as highly hydrophobic glycoproteins (Miller, 2002). The 10 different FZDs encoded in the mammalian genome belong to the superfamily of seven transmembrane-spanning receptors and have been included in the International Union of Basic and Clinical Pharmacology list of G-protein-coupled receptors (GPCRs) as a separate group, the Frizzled receptors, together with the closely related Smoothened receptor (Schulte, 2010). In addition to the FZDs, single-pass transmembrane proteins, in particular LRP5/6 (low-density lipoprotein receptor-related protein), but also ROR2 and RYK have been identified as WNT (co)receptors (Wang et al., 2006; Kikuchi et al., 2009).

There is some experimental evidence that WNTs and FZDs might activate in addition to the 'canonical' $\beta$-catenindependent pathway (Figure 1A), also at least three 'noncanonical', i.e., $\beta$-catenin-independent signaling cascades: the NFAT (nuclear factor of activated T cells)-, the AP-1 (activator protein 1)- and the CRE (cyclic adenosine monophosphate response element)-signaling cascade, as shown in Figure 1B.

Although several studies about certain combinations of WNTs with FZDs, which stimulated either the 'canonical' or the 'non-canonical' pathways, or even both together, have been published (Holmen et al., 2002; Mikels and Nusse, 2006; Verkaar et al., 2008), little information is available on the basic principles of WNT/FZD/coreceptor combinations with regard to the specific activation of the different signaling pathways. This is in part due to the lack of biologically active affordable preparations of most WNTs, and in part due to the sheer number of possible combinations of WNT/FZD/coreceptor, which requires a highly efficient experimental approach to examine in detail even only a preselected part of them.

Here, we demonstrate for the first time that combining the approach of WNT/FZD fusion constructs with the generation of reporter cell lines in HEK 293 cells that permit the use of the highly efficient Flp-In T-REx system for additional stable transfections will allow the investigation of the functional specificity of larger combinations of WNTs, FZDs and coreceptors for the activation of the different pathways. 


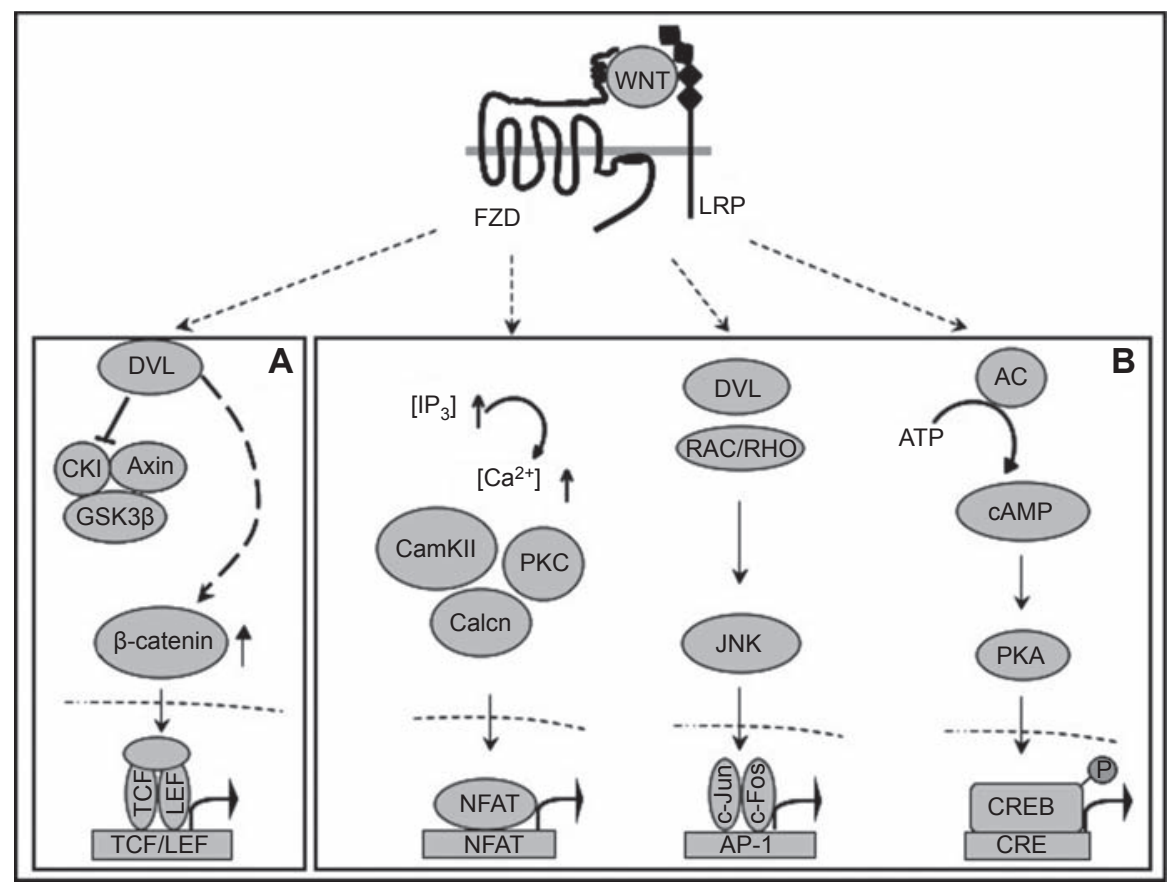

Figure 1 The four major signaling cascades as WNT/FZD/LRP activated pathways.

(A) The 'canonical' $\beta$-catenin pathway. (B) The three 'non-canonical' pathways: NFAT, AP-1 and CRE signaling (from left to right). Abbreviations: FZD, Frizzled receptor; LRP, low-density lipoprotein receptor-related protein; DVL, disheveled; CKI, casein kinase 1; GSK3 $\beta$, glycogen synthase kinase $3 \beta$; TCF, T cell factor; LEF, lymphoid-enhanced factor; $\mathrm{IP}_{3}$, inositol triphosphate; CamKII, calcium/calmodulin dependent protein kinase II; PLC, protein kinase C; Calcn, calcineurin; JNK, Jun N-terminal kinase; AP-1, activator protein 1; AC, adenylylcyclase; cAMP, cyclic adenosine monophosphate; PKA, protein kinase A; CREB, cAMP response element-binding protein; CRE, cAMP response element.

\section{Results}

\section{Functionality of HEK 293 reporter cells for studying receptor-stimulated downstream signaling}

All four different reporter gene plasmids carrying the selection marker geneticin (G418) were stably transfected into Flp-In T-REx HEK 293 cells (referred to hereafter only as HEK 293 cells), and clones with random plasmid integration were selected by addition of G418. Using the HEK 293 cells as the host cell line for the reporter gene constructs enabled us to stably transfect these cells a second time with a geneof-interest using Flp-In technology (Invitrogen, Darmstadt, Germany). Hereby, the plasmid carrying the gene-of-interest becomes integrated with high efficiency through the action of the transiently cotransfected recombinase pOG44, always at the same unique position, where the genome of the host cell line has been modified with a target sequence for pOG44. Moreover, as these HEK 293 cells also constitutively produce a tetracycline-regulatable repressor protein, employing the pcDNA5/FRT/TO vector (Invitrogen) as the expression vector, it is possible to generate transiently or stably transfected cells that express the gene-of-interest only after addition of tetracycline.

A general scheme of the reporter gene constructs is depicted in Figure 2A. The backbone of all reporter constructs was the pcDNA3.1 (Invitrogen) with G418 resistance and the sequence coding for Gaussia luciferase ligated into the multiple cloning site. For each reporter construct, multiple transcriptional response elements as indicated for the respective signaling cascade were inserted $5^{\prime}$ of the TATA box. HEK 293 cells transfected with these reporter gene constructs were selected with G418 and clones for each construct were first tested for functionality with an appropriate transfection-independent control stimulus ( $\mathrm{LiCl}, \mathrm{PMA} /$ ionomycin, PMA, forskolin). Three independent clones with a good signal-to-noise ratio were further propagated and examined in more detail.

The $\beta$-catenin signaling pathway (Figure 1A) is so far the best-understood WNT/FZD cascade. In the absence of WNT proteins, cytoplasmic levels of $\beta$-catenin are kept low, as it is constitutively marked by a destruction complex, consisting of glycogen synthase kinase $3 \beta$ (GSK3 $\beta$ ), axin and adenomatous polyposis coli, for proteasomal degradation. Activation of a FZD by a WNT results in the formation of a ternary WNT/ FZD/LRP complex that recruits axin to the plasma membrane, thereby inhibiting the destruction complex. Consequently, $\beta$-catenin accumulates in the cytoplasm and translocates into the nucleus, where it recruits transcription factors, such as $\mathrm{T}$ cell factor/lymphoid-enhanced factor (TCF/LEF), thus upregulating the transcription of WNT target genes (Logan and Nusse, 2004; Macdonald et al., 2007), e.g., cyclin D1, matrix metalloproteinases, c-myc, etc., or the Gaussia luciferase in our approach. To determine the functionality of our TCF/LEF 

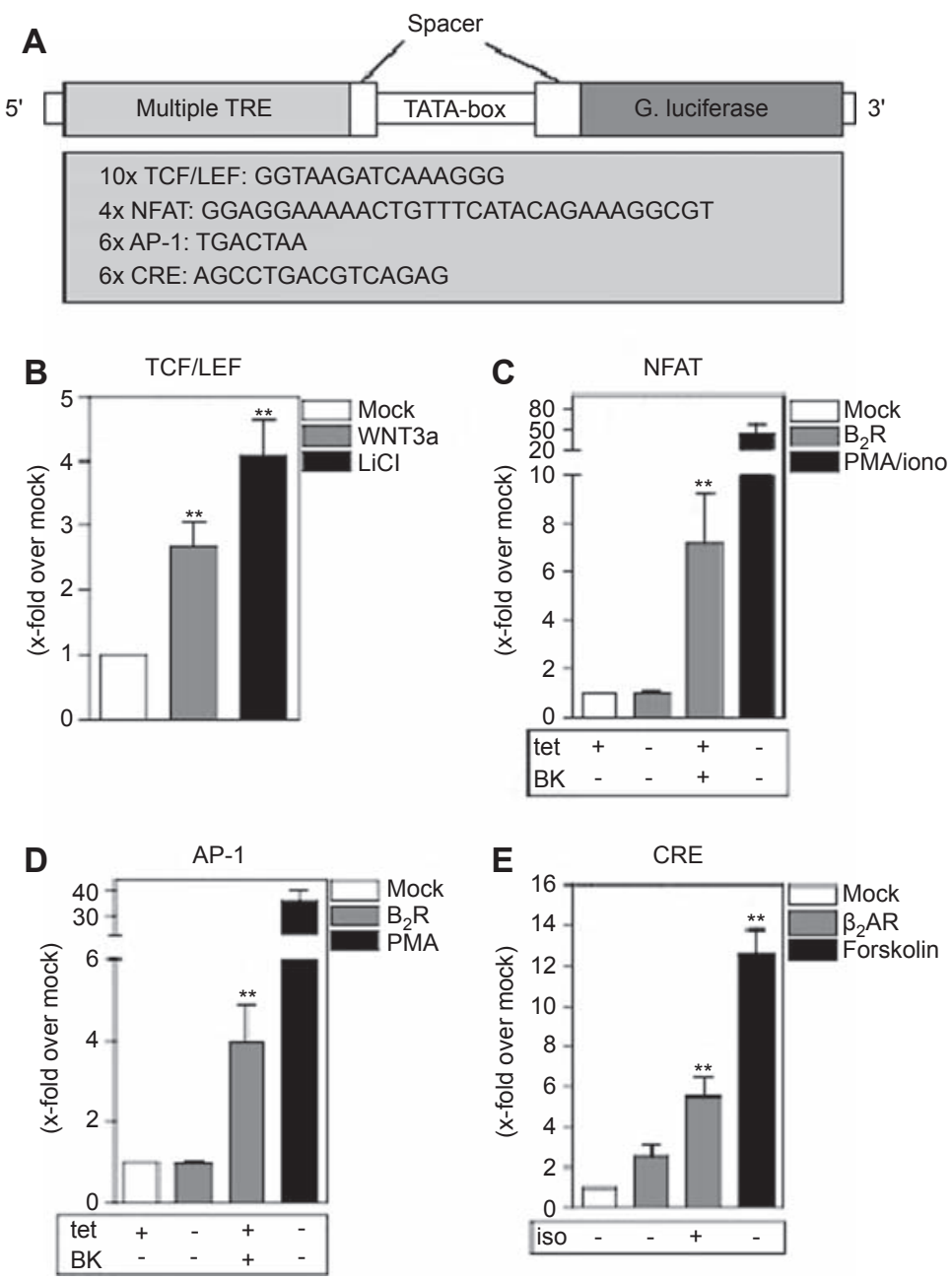

Figure 2 Design and functionality of reporter gene HEK 293 cells.

(A) Design of reporter gene constructs, consisting of specific (TCF/LEF, NFAT, AP-1, CRE) multiple transcriptional response elements (TREs), TATA box and the Gaussia luciferase (G. luciferase) coding sequence. (B) Three independent clones of TCF/LEF reporter HEK 293 cells were transiently transfected with empty vector (mock) or WNT3a plasmid; $10 \mathrm{~mm} \mathrm{LiCl}$ was used as transfection-independent positive control for TCF/LEF inducible Gaussia luciferase expression. (C) Three independent clones of NFAT reporter HEK 293 cells were transiently transfected with mock or bradykinin (BK) $\mathrm{B}_{2}$ receptor $\left(\mathrm{B}_{2} \mathrm{R}\right)$ and stimulated with $1 \mu \mathrm{M} \mathrm{BK} ; 1 \mu \mathrm{M} \mathrm{PMA} / 1 \mu \mathrm{M}$ ionomycin (iono) was used as transfectionindependent positive control. (D) Three independent clones of AP-1 reporter HEK 293 cells were transiently transfected with mock or $\mathrm{B}_{2} \mathrm{R}$ and stimulated with $1 \mu \mathrm{M} \mathrm{BK} ; 1 \mu \mathrm{M}$ PMA was used as transfection-independent positive control. (E) Three independent CRE reporter HEK 293 cells were transiently transfected with mock or $\beta_{2}$-adrenergic receptor $\left(\beta_{2}-\mathrm{AR}\right)$ and stimulated with $10 \mu \mathrm{M}$ isoproterenol (iso); $5 \mu \mathrm{M}$ forskolin was used as transfection-independent positive control. Where indicated expression was induced with $0.5 \mu \mathrm{g} / \mathrm{ml}$ tetracycline (tet). Gaussia luciferase activity was measured and normalized for mock transfected cells. The results are presented as fold increase over mock transfected cells and represent means \pm SEM of at least with three different clones, performed in triplicate. One-way ANOVA with the Dunnett's test: $* * p<0.01$.

reporter HEK 293 cells, we first used the transfection-independent stimulus $\mathrm{LiCl}$, which inhibits GSK3 $\beta$ in the degradation complex and thus promotes the accumulation of $\beta$-catenin. Addition of $\mathrm{LiCl}$ resulted in an increase of Gaussia luciferase activity of approximately 4-fold over mock level (Figure 2B). After this verification of the functionality of the reporter system, we then used the prominent WNT3a as a stimulus. WNT3a is commonly known as a typical 'canonical' WNT protein, because it almost exclusively activates the $\beta$-catenin-mediated pathway (Shimizu et al., 1997). Therefore, as further proof of functionality, we transiently transfected the TCF/LEF reporter cells with a human WNT3a construct and observed the induction of Gaussia luciferase activity approximately 3-fold over mock level (Figure 2B). These results demonstrate that the selected three independent TCF/LEF reporter cells were fully functional, exhibiting a good signal-to-noise ratio, and could be stimulated through transfection with an expression vector, harboring the coding sequence of a WNT protein.

Next, we studied the non-canonical, $\mathrm{Ca}^{2+}$-dependent NFAT cascade. When the level of intracellular $\mathrm{Ca}^{2+}$ is significantly increased, it binds and activates calmodulin (CaM), 
which results in the activation of proteins, such as $\mathrm{Ca}^{2+} / \mathrm{CaM}$ dependent protein kinase II (CamKII), calcineurin (Caln) and protein kinase $\mathrm{C}$ (PKC) (Figure 1B). Activation of Caln leads to the dephosphorylation of the transcription factor NFAT (Berridge et al., 2003), which consequently translocates into the nucleus to induce NFAT-regulated gene expression. To test the generated NFAT HEK 293 reporter cells, we used the $\mathrm{Ca}^{2+}$-ionophore ionomycin in combination with the phorbol ester PMA, a strong PKC activator, as a transfection-independent stimulation. Addition of these reagents to the HEK 293 cells increased NFAT reporter activity approximately 50 -fold over mock level (Figure 2C). To further prove the functionality of our reporter system, we tested the transfection-dependent $\mathrm{B}_{2} \mathrm{R} / \mathrm{BK}$ stimulation. The bradykinin (BK) $\mathrm{B}_{2}$ receptor $\left(\mathrm{B}_{2} \mathrm{R}\right)$ is a $\mathrm{G \alpha} \alpha_{\mathrm{q} / 11}$ (and $\mathrm{G} \alpha_{\mathrm{i}}$ ) coupled GPCR that elicits an increase in intracellular $\mathrm{Ca}^{2+}$ concentrations through release of inositol trisphosphate via activation of phospholipase $\beta$ (Leeb-Lundberg et al., 2005). Transient transfection with $\mathrm{B}_{2} \mathrm{R}$ followed by stimulation with BK produced an increase of the reporter Gaussia luciferase activity by approximately 7 -fold over mock level (Figure $2 C)$. This activity was only observed, when expression of the transiently transfected $\mathrm{B}_{2} \mathrm{R}$ was induced by addition of tetracycline, proving that the inducible T-REx expression system in the three independent clones of HEK 293 reporter cell line is working (Figure 2C). Thus, we could demonstrate the functionality of our NFAT reporter cells with transfectionindependent (ionomycin/PMA) and transfection-dependent $\left(\mathrm{B}_{2} \mathrm{R} / \mathrm{BK}\right)$ stimulation.

As a further non-canonical WNT pathway, we investigated AP-1-dependent gene expression. Activation of the AP-1 promoter can be mediated by more than one signaling pathway that all result in the binding of a dimeric complex of the transcription factors c-Jun and c-Fos to the AP-1 promoter sequence. To test our AP-1 HEK 293 reporter cells, we used PMA as a transfection-independent stimulus that functions mainly through activation of $\mathrm{PKC}$ on the AP-1 promoter. Addition of PMA generated a 40-fold increase of Gaussia luciferase activity over mock level (Figure 2D). As transfection control, we used the $\mathrm{B}_{2} \mathrm{R}$ plasmid because $\mathrm{B}_{2} \mathrm{R}$ also mediates the upregulation of the transcription factor c-Fos via ERK1/2 activation (El-Dahr et al., 1998). Newly synthesized c-Fos consequently dimerizes with c-Jun proteins and forms AP-1 promoter activating complexes. Indeed, AP-1 reporter cells, transiently expressing the $\mathrm{B}_{2} \mathrm{R}$, responded to a challenge with BK with an increase in Gaussia luciferase activity (approximately 4-fold over mock level). No upregulation of Gaussia luciferase activity could be observed without stimulation with $\mathrm{BK}$ or in the absence of $\mathrm{B}_{2} \mathrm{R}$ (when not induced with tetracycline) (Figure 2D). These results show that the three independent clones of AP-1 reporter cells are functional and might serve to identify WNT/FZD/LRP-induced AP-1 promoter activation that according to experimental evidence is most probably mediated via dishevelled (DVL) and activation of JNK (Figure 1B). PMA as a transfection-independent control for NFAT- and AP-1-reporter cells was not tolerated well by the cells resulting in strong responses, and also with larger standard errors. As these errors were not due to the luciferase reporter assay per se, the PMA data were excluded in the statistical calculation.

Finally, we also considered the CRE-regulated non-canonical pathway. Activation of adenylate cyclase leads to the generation of the second messenger cAMP, resulting in the stimulation of protein kinase A (PKA), which phosphorylates and thus activates transcription factors, such as CRE-binding proteins, which increases cAMP-mediated gene expression (Figure 1B) (Karin and Hunter, 1995). As a transfection-independent reagent, we used forskolin, a direct activator of adenylyl cyclase, which led to an increase of Gaussia luciferase activity in the CRE reporter HEK 293 cells of approximately 12 -fold over mock (Figure 2E). To further confirm the functionality, we tested our approach with the $\beta_{2}$-adrenergic receptor $\left(\beta_{2} \mathrm{AR}\right)$, which is the prototype of GPCRs that activate adenylate cyclase via $\mathrm{G}$ protein $\mathrm{G} \alpha_{\mathrm{s}}$ (Lefkowitz and Caron, 1988). Stimulation of transiently overexpressed $\beta_{2}$ AR with the agonist isoproterenol increased Gaussia luciferase activity in the CRE HEK 293 reporter cell line by approximately 5 -fold over mock level (Figure 2E). In the absence of isoproterenol, no significant increase in Gaussia luciferase activity was observed, proving the specificity of the CRE-mediated response in this reporter cell line.

\section{Fusion proteins as a tool for studying WNT/FZD signaling}

Holmen et al. (2002) have shown that some WNT/FZD fusion proteins are functional with regard to the $\beta$-catenin pathway by generating chimera of Xenopus WNT8 and mouse or human FZDs and expressing them either in Xenopus eggs or in HEK 293T cells. We set out to pursue this fusion approach with human WNT/FZD chimera in our reporter gene HEK 293 cells, to determine the signaling specificity of different WNT/FZD/LRP combinations. As proof-of-principle that these WNT/FZD fusion proteins will be functional in our reporter gene cells, we first generated a WNT3a/FZD4 chimera. We decided to begin with human WNT3a because it is one of the best-investigated WNTs and functionally active WNT3a protein is commercially available for control experiments. FZD4 was selected because it has been reported that it can activate canonical and non-canonical pathways (Mikels and Nusse, 2006).

Lacking a specific FZD4 antibody, for control experiments, we generated a FZD4 expression construct with a combined hemagglutinin- and myc-epitope tag inserted immediately after the FZD4 signal peptide (Figure 3A). This allowed expression of the receptor on the HEK 293 cell surface with the tag still intact after proper processing of the signal peptide. For the construction of the WNT3a/FZD4 chimera (termed WNT3aFZD4), we closely followed the approach of Holmen et al. (2002). The sequence of WNT3a containing the original WNT3a signal peptide at its N terminus was followed by a polyglycine spacer and the myc-tagged FZD4 sequence, lacking the FZD4 signal peptide (Figure 3B). The entire construct was ligated into the pcDNA5/FRT/TO vector, whereby its expression in our HEK 293 cells depends on the addition of tetracycline. 

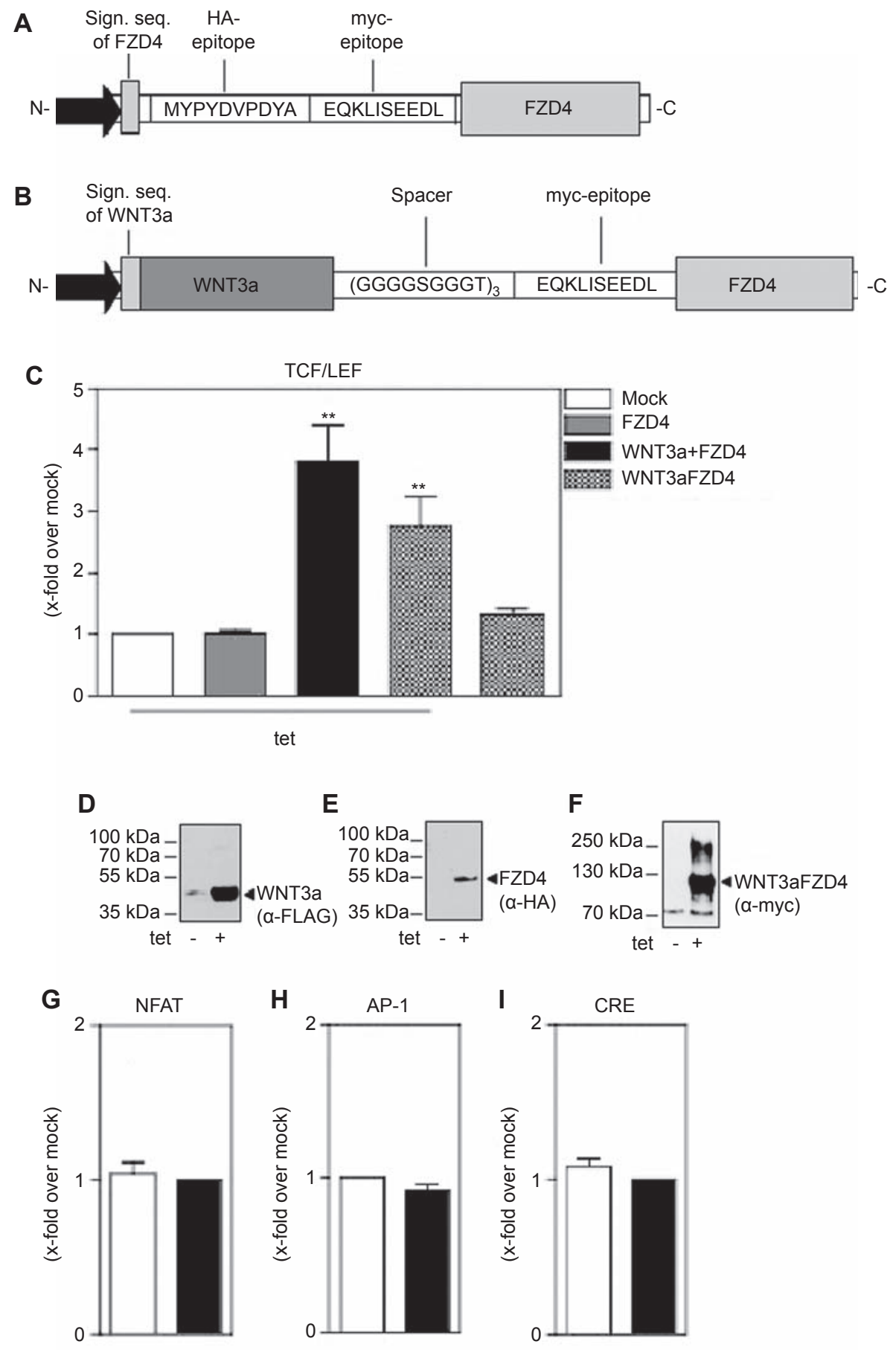

Mock

WNT3aFZD4

Figure 3 Design and functionality of FZD4 construct and chimera WNT3aFZD4.

(A) Schematics of the tagged FZD4 receptor. The signal sequence of FZD4 was followed by a double tag [hemagglutinin (HA)- and mycepitope] and the remaining FZD4 protein. (B) Schematics of the WNT3aFZD4 fusion protein. WNT3a (containing its signal peptide, sign. seq.) was fused via a glycine-rich spacer and a myc-epitope to the sequence of FZD4. (C) Three independent clones of TCF/LEF reporter HEK 293 cells were transiently transfected with FZD4, FZD4 and WNT3a, or fusion construct WNT3aFZD4, induced or not with tetracycline (tet). (D) Anti-FLAG immunoblot of transiently transfected WNT3a with or without tetracycline (tet) induction. (E) Anti-HA immunoblot of transiently transfected FZD4 plasmid with or without tetracycline (tet) induction. (F) Anti-myc immunoblot of transiently transfected WNT3aFZD4 with or without tetracycline (tet) induction. (G-I) Three independent clones of NFAT-, AP-1- and CRE-reporter HEK 293 cells were transiently transfected with empty vector (mock) or WNT3aFZD4. Gaussia luciferase activity was determined as described in the Materials and methods section. The results are presented as fold increase over mock transfected calls and represent means \pm SEM of at least four independent experiments with three different clones, performed in triplicate. One-way ANOVA with the Dunnett's test: ** $p<0.01$. 
When the expression of the fusion construct WNT3aFZD4 after transient transfection into TCF/LEF reporter cells was induced by tetracycline, its functionality was verified by the 3-fold increase in Gaussia luciferase activity over mock transfection (Figure 3C). Cotransfection with WNT3a and FZD4 plasmids resulted in a 5-fold upregulation of Gaussia luciferase activity, which was similar to the transfection with the WNT3a plasmid alone (Figure 2B). This suggests that the effect of the cotransfected plasmids is almost exclusively due to WNT3a acting via endogenous receptors, and not via the cotransfected FZD4.

Immunoblots confirmed that all transiently transfected constructs were well expressed (Figure 3D-F), with the blot of WNT3aFZD4 displaying in addition a tetracycline-inducible band at double the predicted size, suggesting the formation of dimers (Figure 3F).

When the fusion protein WNT3aFZD4 was transiently transfected in each of the other three 'non-canonical' reporter cell lines (NFAT-, AP-1- and CRE-reporter HEK 293 cells), no increased Gaussia luciferase activity was observed compared with mock transfection (Figure 3G-I), indicating that WNT3aFZD4 stimulates only the 'canonical' $\beta$-catenin pathway. Alternatively, signaling via these 'non-canonical' pathways might require additional cellular components, e.g., LRPs, which are probably not available in the necessary amounts in these HEK 293 cells, unless being overexpressed.

\section{Enhancement of WNT3aFZD4 response in TCF/LEF reporter by LRP6}

The receptors LRP5 and LRP6 are large single-pass membrane proteins, characterized by an extended extracellular cysteinerich domain and a small cytoplasmic carboxy-terminal domain.
They are commonly known as FZD coreceptors as they have been shown to play an essential role in the activation of the $\beta$-catenin signaling cascade via WNTs bound to FZDs (Figure 1A). To investigate a potential specific role of at least one of the two LRPs in the activation of the $\beta$-catenin signaling cascade, we stably transfected LRP6 in our TCF/LEF reporter cells using their still available Flp-In site.

The TCF/LEF reporter cell line stably and inducibly expressing the LRP6 receptor (Figure 4B on mRNA level and Figure $4 \mathrm{C}$ on protein level) displayed basal reporter gene activity after induction with tetracycline that was twice as high as the one observed in the absence of LRP6 overexpression (data not shown), suggesting an enhancement of endogenous WNT/FZD signaling by the overexpressed LRP6. We transfected the TCF/LEF HEK 293 cell line transiently with WNT3a and FZD4 plasmids either alone or in combination. Whereas FZD4 alone did not induce an increase in Gaussia luciferase activity without overexpression of LRP6 (Figure 3C), in the LPR6-overexpressing TCF/LEF reporter cells FZD4 enhanced the reporter gene activity 4-fold over mock transfected cells (Figure 4A). Overexpression of WNT3a alone resulted in an activity increase of approximately 6-fold over mock level similar to the one observed for TCF/LEF cells without LRP6 overexpression (Figure 3C). However, cotransfection of WNT3a and FZD4 into the TCF/LEF-LRP6 reporter cells could activate luciferase activity by approximately 15 -fold (Figure 4A), thus displaying a clearly synergistic effect, which was not seen in TCF/LEF reporter cells without coreceptor overexpression (Figure 3C).

The highest activities suggesting a potential function of the coreceptor LRP6 were determined after transfection of the fusion construct WNT3aFZD4 into the TCF/LEF-LRP6 reporter cells (Figure 4A). The 35-fold higher induction of
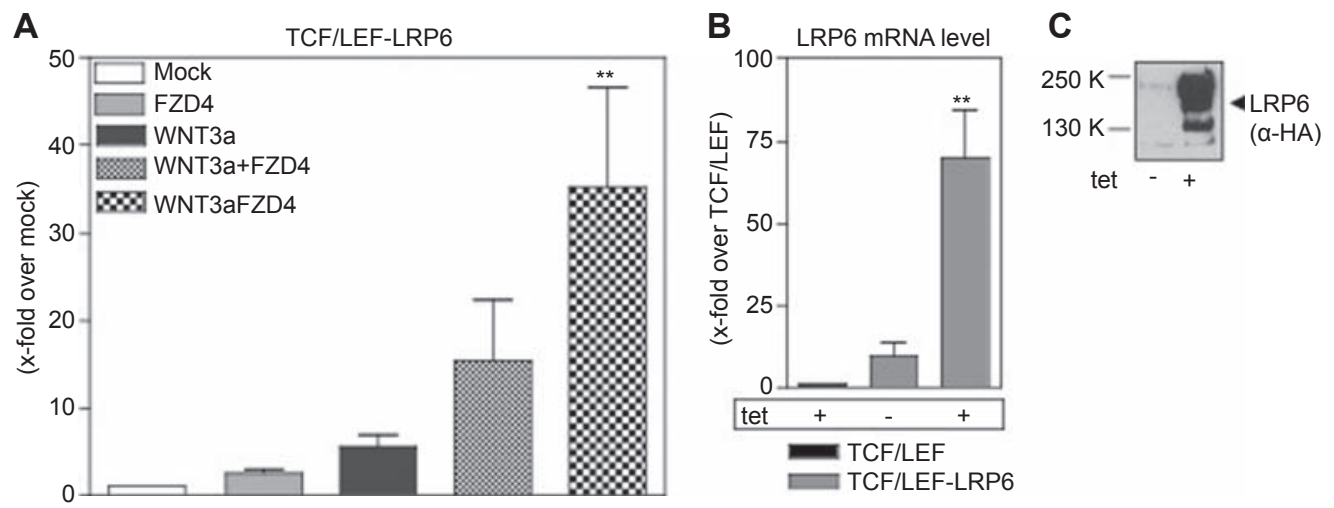

Figure 4 Gaussia luciferase activity in TCF/LEF reporter HEK 293 cells stably expressing LRP6.

(A) Representative clone of TCF/LEF reporter HEK 293 cells stably expressing LRP6 was transiently transfected with empty vector (mock), FZD4, WNT3a (separately or together), or with the fusion construct WNT3aFZD4, and expression induced with tetracycline; $48 \mathrm{~h}$ post-transfection Gaussia luciferase activity was measured and normalized for mock transfected cells. (B) Endogenous mRNA expression levels in TCF/ LEF reporter HEK 293 cells, compared with TCF/LEF reporter HEK 293 cells stably expressing LRP6; 48 h post-induction with tetracycline (tet), RNA was isolated, transcribed to cDNA and qPCR with specific LRP6 primers was performed. (C) Representative anti-HA immunoblot of TCF/LEF reporter HEK 293 cells stably expressing LRP6 with and without tetracycline (tet) induction (approx. $20 \mu \mathrm{g}$ protein/lane). Values represent means \pm SEM of three independent experiments for Gaussia luciferase measurements and three independent experiments for endogenous mRNA level expression, performed in triplicate. One-way ANOVA with the Dunnett's test: ** $p<0.01$. 
the Gaussia luciferase activity over mock, compared with the only 3-fold induction in TCF/LEF reporter cells (Figure 3C), points to a strong productive interaction of the coreceptor LRP6 with the WNT3aFZD4 fusion protein in the stimulation of the $\beta$-catenin signaling pathway.

\section{Discussion}

Important unsolved questions in the field of WNT signal transduction are as follows. What are the molecular mechanisms for the specificity of WNT/FZD signaling? What signaling pathways - $\mathrm{G}$ protein independent or dependent - become activated? Are specific WNT/FZD combinations required to stimulate the different signaling pathways (Kikuchi et al., 2007; van Amerongen et al., 2008; Grumolato et al., 2010)? A major reason for this lack of knowledge lies in the high numbers of WNTs and FZDs giving rise to many possible combinations, which even increase when additional membrane receptors, such as the coreceptors LRP5 or 6 are taken into account as (potential) signaling participants. Moreover, the poor availability of functional preparations of most WNTs often hindered a successful experimental strategy to address these open questions.

Therefore, in our study we pursued the following experimental approach: (i) random integration of reporter gene plasmids into HEK 293 cells carrying the Flp-In T-REx293 system that allows an additional site-directed stable and inducible transfection and also works for large genes, such as the LRP6; (ii) construction and transient or stable transfection of WNT/FZD fusion proteins closely designed following the approach of Holmen et al. (2002).

We could demonstrate here that our four reporter cell lines with the Gaussia luciferase gene under control of promoters specific for the respective pathways (the canonical $\beta$-catenin and the non-canonical NFAT, AP-1 and CRE pathways) can be activated by appropriate transfection-dependent and transfection-independent stimuli. The observed activation of the Gaussia luciferase expression cannot be an artifact caused by direct effects of the treatment on cell number or viability, as no significant differences were found between the various samples (with the exception of the PMA treated cells, as indicated) when cell numbers were measured in the preliminary experiments (data not shown). Variability between the experimental series was so low that it was considered appropriate to combine the data normalized to the mock cells in each experiment. No further normalization to cell number or cell viability was assumed to be required. Furthermore, as demonstrated as proof-of-principle by the WNT3aFZD4 chimeric construct without and with coexpression of LRP6, the use of different WNT/FZD fusion constructs should allow the investigation of various specific interactions between WNTs, FZDs and coreceptors in the described reporter cells.

Because the fusion protein has to be newly synthesized by the reporter cells before it can elicit a response, it is apparent that the fusion protein approach is suitable only to determine the long-term effects of longer lasting receptor stimulation. This approach is, therefore, most useful for the read- out via reporter gene assays but not for assays that measure fast responses, such as the inositol trisphosphate release or increase of intracellular $\mathrm{Ca}^{2+}$. However, as most WNT/FZDmediated long-term responses involve activation of transcription factors and gene expression, the fusion protein approach is somewhat reasonable to elucidate their signaling specificities. Moreover, it offers the important advantage that a specific WNT is joined covalently to a FZD and therefore most likely can act only via this bound FZD (and apparently the necessary coreceptors; Figure 4A) but not via other FZDs endogenously expressed in the transfected cells. The latter fact always has to be considered because many cells do express FZDs and are needed for many developmental and physiological processes. As the stimulatory effect of the (over)expression of WNT3a on the $\beta$-catenin pathway without additional overexpression of a FZD is demonstrated (Figure 2B), this is apparently also true for HEK 293 cells. Therefore, the approach using specific WNT/FZD fusion constructs will render an easier interpretation of the obtained results, as most likely no competitive signaling - whether inhibiting or increasing - by endogenous FZDs will have to be considered. In this context, it should be emphasized that the immunoblot of WNT3aFZD4 revealed only the intact fusion protein (or twice its size; Figure $3 \mathrm{~F}$ ) and hardly any FZD4 that might have lost WNT3a through shedding on the cell surface.

Furthermore, the WNT3aFZD4 fusion protein was activating the $\beta$-catenin signaling cascade but not the other "noncanonical' NFAT, AP-1 and CRE pathways. However, taking into account the strong effect that the coexpression of LRP6 had on the response of the $\beta$-catenin pathway (Figure 4A), future experiments will have to show whether this lack of a response in the non-canonical pathways is an intrinsic property of the WNT3a/FZD4 combination or whether potentially necessary components (e.g., LRPs) were missing in the HEK 293 cells. Further experiments, performed in the meantime, suggest that even the prototypical 'non-canonical' ligand WNT5a (fused to FZD4) is not able to activate any of the 'non-canonical' reporter cells (data not shown). Our set-up, as shown as proof-of-principle, should allow us to determine in future experiments whether this is due to the particular WNT/ Frizzled receptor combination or to missing cellular components. Taken together, our results clearly demonstrate that the combination of reporter gene constructs in Flp-In T-Rex HEK 293 cells with the expression of WNT/FZD fusion proteins should provide the tools to identify WNTs, FZDs and/or coreceptors and their specific interaction for the stimulation of 'canonical' or 'non-canonical' WNT pathways in an experimentally feasible way.

\section{Materials and methods}

\section{Materials}

Flp-In T-REx-293 HEK 293 cells were purchased from Invitrogen. A vector carrying the $\beta_{2}$-adrenergic receptor was kindly provided by Prof. M. Lohse (University of Würzburg, Würzburg, Germany). The FZD4 coding sequence was purchased from the Missouri S\&T cDNA 
Resource Center (Rolla, MO, USA). EcoTransfect was obtained from OzBiosciences (Marseille, France). Poly-D-lysine was purchased from Sigma-Aldrich (Taufkirchen, Germany). Cell culture media and additions were obtained from PAA Laboratories (Coelbe, Germany). Coelenterazin was purchased from PJK (Kleinblittersdorf, Germany). FuGene HD reagent and EDTA-free protease inhibitor tablets were obtained from Roche (Mannheim, Germany). G418-BC sulfate was from Biochrom AG (Berlin, Germany). Ionomycin (A23187, free acid) and Phorbol 12-myristate-13-acetate (PMA) were obtained from Calbiochem (Darmstadt, Germany). Oligonucleotides were synthesized by Metabion (Martinsried, Germany) or MWG-Biotech (Ebersberg, Germany).

\section{Reporter gene plasmids}

Using standard PCR techniques and appropriate primers, including the required restriction sites (sense: 5'-AATTAAGCTTATG GGAGTCAAAGTTCTGTTTGC-3'; antisense: 5'-GATCAAG GGGGCCGGTGGTGACTAACTCGAGATTATT-3'), the coding sequence of Gaussia luciferase (GLuci) was amplified from the pGLuc-Basic vector (New England Biolabs, Ipswich, MA, USA) and cloned between the HindIII/XhoI sites of the pcDNA3.1 expression vector (Invitrogen). Thereafter, the NFAT promoter sequence harboring four NFAT transcription response elements (GGAGGAAAAACTGTTTCATACAGAAAGGCGT) and the TATA box were amplified using appropriate primers (sense: 5'-TTAATTCAATTGCTTATCATGTCT-3'; antisense: 5'GCATTCCGGTACTGTTGGTAAGCTTTAAT- ${ }^{\prime}$ ) and the pHTSNFAT plasmid (Biomyx, San Diego, CA, USA) as template. The resulting product was ligated into the MfeI/HindIII sites of the generated pcDNA3.1-GLuci vector, giving the NFAT reporter gene plasmid. A plasmid synthesized by Genart (Regensburg, Germany) harboring the TCF/LEF promoter sequence with 10 multiple transcription response elements (GGTAAGATCAAAGGG), the TATA box, a 5'-MfeI site, and a 3'-HindIII site, was digested with MfeI/ HindIII and the entire sequence ligated into the correspondingly digested NFAT reporter gene vector, giving the TCF/LEF reporter gene plasmid. The AP-1 multiple transcriptional response element with six TGACTAA repeats was constructed by standard PCR techniques using suitably designed sense (5'-CATGTCTGCGGCCGCAATGACTAATGACTAATGACTAATGACTAATGACTAAGCGGAGACTCTAGAGG-3') and antisense (5'-GCGGAGACTCTAGAGGGTATATAATGGAAGCTCGAATTCCAGCTTGGCATTCCGGTACTGTTGGTAAGCTTTAAT-3') primers also introducing 5'-MfeI and 3'-HindIII restriction sites, and subcloned into the NFAT reporter plasmid. The CRE multiple transcriptional response element with four AGCCTGACGTCAGAG repeats was generated by standard PCR techniques and appropriate primers (sense: 5'-GTCTGCGGCCGCAAGCCTGACGTCAGAGAGCCTGACGTCAGAGAGCCTGACGTCAGAGAGCCTGACGTCAGAGCGGAGACTC-3'; antisense: 5'-GTCAGAGCGGAGACTCTAGAGGGTATATAATGGAAGCTCGAATTCCAGCTT-3') introducing $5^{\prime}$-NotI and $3^{\prime}$-EcoRI restriction sites, and subcloned into the NotI/EcoRI digested AP-1 reporter plasmid.

\section{Expression vectors}

A combined hemagglutinin (HA) and myc tag was introduced by standard PCR techniques with the following primers (sense: $5^{\prime}$ AATTGCGGCCGCATTCGGGGACGAGGAAGAGCG-3'; antisense: 5'-AAATTTCTCGAGTTATACCACAGTCTCACTGC-3') after the signal peptide coding sequence of the human FZD4 and the entire construct subcloned into the pcDNA5/FRT/TO plasmid.
For generation of the fusion construct WNT3aFZD4, the WNT3a coding sequence without the stop codon, followed by a spacer, a myc-epitope, and the FZD4 coding sequence was generated and subcloned into the pcDNA5/FRT/TO plasmid using standard PCR techniques with appropriate primers (sense: $5^{\prime}$-AATTAAGCTTATGGCCCCACTCGGATACTTC-3'; antisense: 5'-TATGGTACCCTTGCAGGTGTGCACGTCGTAGAC-3') and templates. For the generation of a C terminally FLAG-tagged WNT3a plasmid, oligonucleotides with NotI/XhoI digestion sites and a sequence coding for a double FLAG epitope with a stop codon, were generated by standard PCR techniques and subcloned into the NotI/XhoI digested WNT3aFZD4 plasmid, thus exchanging the FZD4 sequence for the double FLAG sequence. The sequence for the LRP6 was amplified from HEK 293 cDNA with corresponding primers using standard PCR techniques, and subcloned into a pcDNA5/FRT/TO plasmid providing a $\mathrm{C}$ terminal HA-tag.

\section{Cell culture and stable transfection}

The HEK 293 cells were cultured in DMEM high glucose supplemented with $10 \%$ fetal calf serum (FCS) and $1 \%$ penicillin/streptomycin. For stable transfection with reporter plasmids, cells in 12-well plates (60\% confluency) were transfected with $1 \mu \mathrm{g}$ DNA and $3 \mu \mathrm{l}$ FuGene reagent according to the manufacturer's manual. Selection was done with G418 (1 mg/ml) and resulted in single stably transfected clones expressing the corresponding reporter plasmids. Three independent clones with low basal levels of Gaussia luciferase activity of each reporter were chosen for further experiments. For stable transfection of HEK 293 cells with pcDNA5/FRT/TO expression vectors, cells on 12 -well plates (60\% confluency) were transfected with a total amount of $2 \mu \mathrm{g}$ DNA (consisting of $0.4 \mu \mathrm{g}$ of the respective pcDNA5/FRT/TO plasmid and $1.6 \mu \mathrm{g}$ pOG44 plasmid) and $6 \mu 1$ FuGene reagent according to the manufacturer's manual. Selection was performed with hygromycin B $(0.5 \mathrm{mg} / \mathrm{ml})$.

\section{Reporter gene assays}

For transient transfections, reporter cells were seeded in 24-well plates coated with poly-D-lysine to improve cell adherence and cultured overnight to gain $70 \%$ confluency. Transfections were performed with EcoTransfect reagent according to the manufacturer's manual with $0.2 \mu \mathrm{g}$ plasmid and $0.4 \mu \mathrm{l}$ transfection reagent. After $24 \mathrm{~h}$, the medium was changed to DMEM with $0.5 \% \mathrm{FCS}, 1 \%$ penicillin/streptomycin and expression was induced by addition of $0.5 \mu \mathrm{g} / \mathrm{ml}$ tetracycline. After another $24 \mathrm{~h}$ of incubation, cells were stimulated with the respective stimulus. Then, $72 \mathrm{~h}$ post-transfection, $75 \mu \mathrm{l}$ supernatant, containing the secreted Gaussia luciferase, was transferred to a black 96-well plate (Corning Incorporated, New York, NY, USA). Upon addition of $25 \mu 1$ Gaussia luciferase substrate [5.72 $\mu \mathrm{m}$ coelenterazin, $2.2 \mathrm{~mm} \mathrm{Na} \mathrm{EDDT}_{2}$, $0.22 \mathrm{M} \mathrm{K}_{\mathrm{x}} \mathrm{PO}_{4}$, $\mathrm{pH} 5.1 ; 0.44 \mathrm{mg} / \mathrm{ml}$ bovine serum albumin (BSA), $1.1 \mathrm{M} \mathrm{NaCl}$, $1.3 \mathrm{~mm} \mathrm{NaN}_{3}, \mathrm{pH} 5.0$ ], activity was monitored within $1 \mathrm{~min}$ in a Transluminator DarkReader TECAN safire2 (Clare Chemical Research, Dolores, CO, USA), followed by quantification using XFluor4 Safire2 software (Tecan, Crailsheim, Germany).

\section{RNA isolation, cDNA synthesis and quantitative real- time PCR (qPCR) analysis}

For analyzing the endogenous level of TCF/LEF reporter HEK 293 cells with and without stably transfected LRP6, cells were seeded at 12 -well plates, coated with poly-D-lysine and induced 
with tetracycline after $24 \mathrm{~h}$ incubation. Then, $48 \mathrm{~h}$ post-induction total RNA was isolated with an RNeasy Mini Kit (Qiagen, Hilden, Germany), according to the manufacturer's manual. Genomic DNA removal and cDNA synthesis of $1 \mu \mathrm{g}$ RNA was completed by using the QuantiTect Reverse Transcription Kit (Qiagen). qPCR was analyzed by preparing a mastermix for a $10 \mu 1$ reaction by mixing: $7 \mu 1$ nuclease-free water, $1 \mu \mathrm{l}$ LightCycler-Primer Set for human LRP6 (Search-LC, Heidelberg, Germany), $1 \mu$ l LightCycler-FastStart DNA Master SYBR Green I Kit (Roche Applied Science, Manheim, Germany) and $1 \mu \mathrm{l}$ of template cDNA with the following thermal cycling conditions on a LightCycler (Roche Applied Science) with LightCycler Software version 3: predenaturation $\left(95^{\circ} \mathrm{C}, 10 \mathrm{~min}\right)$; 40 cycles of repeated denaturation $\left(95^{\circ} \mathrm{C}\right.$ for $\left.10 \mathrm{~min}\right)$; annealing of primers $\left(68-58^{\circ} \mathrm{C}, 0.5^{\circ} \mathrm{C} / \mathrm{s}, 10 \mathrm{~min}\right)$ and elongation $\left(72^{\circ} \mathrm{C}, 16 \mathrm{~min}\right)$. At the end of the run an additional melt curve step was included from $58^{\circ} \mathrm{C}$ to $95^{\circ} \mathrm{C}$, rising $0.5^{\circ} \mathrm{C} / \mathrm{s}$.

\section{Immunoblot analysis}

After determination of Gaussia luciferase activity in the supernatant, reporter gene cells were lysed in $60 \mu \mathrm{l}$ cell lysis buffer $(10 \mathrm{~mm}$ Tris- $\mathrm{HCl}$, $25 \mathrm{~mm} \mathrm{KCl}, 150 \mathrm{~mm} \mathrm{NaCl}, 0.1 \%$ Triton X-100, pH 7.4) containing a protease inhibitor cocktail (Roche). For immunoblot analysis proteins in a $40 \mu \mathrm{l}$ aliquot of a 1:1 mixture of lysate and loading buffer [0.2 M DTT, $2 \times$ LDS sample buffer (Invitrogen)] were separated by SDS-PAGE and blotted on nitrocellulose membranes. After $1 \mathrm{~h}$ of incubation in blocking buffer consisting of 5\% (w/v) nonfat dry milk in TBST (50 mM Trisbuffered saline, $150 \mathrm{~mm} \mathrm{NaCl}, 0.1 \%$ Tween 20, pH 7.5) membranes were further incubated for $60 \mathrm{~min}$ with the respective antibody. For HA-tagged receptor FZD4 and LRP6 the membranes were incubated with anti-HA-HRP (1:2000) (Roche, 11867423001) in blocking buffer. The FLAG-tagged WNT3a plasmid was detected with anti-FLAG (1:1000) (F921, Sigma) antibody. For detection of the myc-tagged fusion construct WNT3aFZD4, $60 \mu$ lysate was incubated with $15 \mu \mathrm{l}$ EZview ${ }^{\mathrm{TM}}$ Red anti-myc affinity matrix (Sigma-Aldrich, Taufkirchen, Germany). After $1 \mathrm{~h}$ incubation at $4^{\circ} \mathrm{C}$, the matrix was sedimented by centrifugation and washed intensively with lysis buffer. Precipitated myc-tagged protein was solubilized by boiling in $15 \mu$ l loading buffer [0.1 м DTT, 2×LDS sample buffer (Invitrogen)], separated on a SDSPAGE, blotted on a nitrocellulose membrane, and incubated with antimyc (9E10) antibody (1:500) (sc-40, Santa Cruz, CA, USA), according to the manufacturer's manual. Blots for FLAG- and myc-tagged samples were additionally incubated with anti-mouse-HRP (1:5000) (7726, Cell Signaling, Danvers, MA, USA) for another hour. After intensive washing of the membranes with TBST, visualization was performed by Chemiluminescence Reagent Plus (Roche) on X-ray hyperchemiluminscence films (Hyperfilm ECL, GE Healthcare, Munich, Germany).

\section{Protein quantification}

Total protein quantification was performed using the Micro BCA Protein assay reagent kit from Pierce (Rockford, IL, USA) with BSA as standard.

\section{Data analysis}

Gaussia luciferase activity was measured in triplicate with three different clones in at least four independent experiments and is presented as mean \pm SEM. All data analysis was performed with GraphPad Prism for Macintosh, version 4.0c (GraphPad Software, Inc., San Diego, CA, USA). One-way analysis of variance (ANOVA) with the
Dunnett's test were employed with $p<0.05$ and $p<0.01$ considered statistically significant.

\section{Acknowledgments}

The authors thank C. Seidl for excellent technical assistance in the generation of various plasmid constructs.

\section{References}

Berridge, M.J., Bootman, M.D., and Roderick, H.L. (2003). Calcium signaling: dynamics, homeostasis and remodelling. Nat. Rev. Mol. Cell Biol. 4, 517-529.

El-Dahr, S.S., Dipp, S., and Baricos, W.H. (1998). Bradykinin stimulates the ERK $\rightarrow$ Elk- $\rightarrow$ Fos/AP-1 pathway in mesangial cells. Am. J. Physiol. 275, F343-F352.

Grumolato, L., Liu, G., Mong, P., Mudbhary, R., Biswas, R., Arroyave, R., Vijayakumar, S., Economides, A.N., and Aaronson, S.A. (2010). Canonical and noncanonical Wnts use a common mechanism to activate completely unrelated coreceptors. Genes Dev. 24, 2517-2530.

Holmen, S.L., Salic, A., Zylstra, C.R., Kirschner, M.W., and Williams, B.O. (2002). A novel set of Wnt-Frizzled fusion proteins identifies receptor components that activate beta-catenin-dependent signaling. J. Biol. Chem. 277, 34727-34735.

Karin, M. and Hunter, T. (1995). Transcriptional control by protein phosphorylation: signal transmission from the cell surface to the nucleus. Curr. Biol. 5, 747-757.

Kikuchi, A., Yamamoto, H., and Kishida, S. (2007). Multiplicity of the interactions of Wnt proteins and their receptors. Cell. Signal. 19, 659-671.

Kikuchi, A., Yamamoto, H., and Sato, A. (2009). Selective activation mechanisms of Wnt signaling pathways. Trends Cell Biol. 19, 119-129.

Klaus, A. and Birchmeier, W. (2008). Wnt signaling and its impact on development and cancer. Nat. Rev. Cancer 8, 387-398.

Leeb-Lundberg, L.M., Marceau, F., Muller-Esterl, W., Pettibone, D.J., and Zuraw, B.L. (2005). International Union of Pharmacology. XLV. Classification of the kinin receptor family: from molecular mechanisms to pathophysiological consequences. Pharmacol. Rev. 57, 27-77.

Lefkowitz, R.J. and Caron, M.G. (1988). Adrenergic receptors. Models for the study of receptors coupled to guanine nucleotide regulatory proteins. J. Biol. Chem. 263, 4993-4996.

Logan, C.Y. and Nusse, R. (2004). The Wnt signaling pathway in development and disease. Annu. Rev. Cell Dev. Biol. 20, 781-810.

Macdonald, B.T., Semenov, M.V., and He, X. (2007). SnapShot: Wnt/ $\beta$-catenin signaling. Cell 131, 1204.

Mikels, A.J. and Nusse, R. (2006). Purified Wnt5a protein activates or inhibits $\beta$-catenin-TCF signaling depending on receptor context. PLoS Biol. 4, 570-582.

Miller, J.R. (2002). The Wnts. Genome Biol. 3, REVIEWS3001.

Schulte, G. (2010). International Union of Basic and Clinical Pharmacology. LXXX. The class Frizzled receptors. Pharmacol. Rev. 62, 632-667.

Shimizu, H., Julius, M.A., Giarre, M., Zheng, Z., Brown, A.M., and Kitajewski, J. (1997). Transformation by Wnt family proteins correlates with regulation of $\beta$-catenin. Cell Growth Differ. 8, 1349-1358.

van Amerongen, R., Mikels, A., and Nusse, R. (2008). Alternative wnt signaling is initiated by distinct receptors. Sci. Signal 1 , re9. 
Verkaar, F., van Rosmalen, J.W., Smits, J.F., Blankesteijn, W.M., and Zaman, G.J. (2008). Stably overexpressed human Frizzled-2 signals through the $\beta$-catenin pathway and does not activate $\mathrm{Ca}^{2+}$ mobilization in human embryonic kidney 293 cells. Cell. Signal. $21,22-33$.
Wang, H.Y., Liu, T., and Malbon, C.C. (2006). Structure-function analysis of Frizzleds. Cell. Signal. 18, 934-941.

Received May 23, 2011; accepted August 21, 2011 